\title{
Study of acute pulmonary embolism patients in Sohag University Hospital
}

\author{
Hassan Ahmed Hassanin, Nayel Abd ELhameed Zaki , Alaa Ahmed \\ Ghaleb, Eslam Ahmed Rabee.
}

Internal Medicine Department, Sohag Faculty of Medicine, Sohag University.

\section{ABSTRACT}

Background: Acute pulmonary embolism (PE) is a common and potentially lethal form of venous thromboembolism (VTE) which is commonly encountered in clinical practice. Most patients die of this fatal condition usually within the first $1 \mathrm{~h}$ of the event with mortality rate reaching nearly $10 \%$ during this period ${ }^{(1)}$

Objective: The objective of this study was to determine of risk factors and clinical presentation of acute pulmonary embolism at Sohag University Hospitals

patients\& Methods: All the patients underwent the following:

(1) Detailed history was taken. (2) Examination: Full clinical examination. (3) Investigations: (ABG, D. Dimer, CBC, ECG, Echocardiography, and CT Angiography).

Results: As regard risk factors of pulmonary embolism $70 \%$ of them have DVT, $42 \%$ have dyslipidemia $32 \%$ were postoperative, $28 \%$ were diabetics, $24 \%$ were hypertensive, $22 \%$ were smokers and $20 \%$ were females taking cocs).

Conclusion: The study show that PE more common in females and the mean age of the studied patients was 45years The most common risk factor is DVT.

Keywords: acute pulmonary embolism

\section{Introduction}

Mortality rate of diagnosed and treated pulmonary embolism ranges from 3 to $8 \%$, but increases to about $30 \%$ in untreated pulmonary embolism. ${ }^{(1)}$ In the United States, acute pulmonary embolism afflicts 500,000- 600,000 persons annually and is either a primary or secondary cause of death in $150,000-200,000$ of these individuals. ${ }^{(2)}$ The 1-year mortality rate in the PIOPED study was reported as $25 \%$, with $2.5 \%$ dying from primary cause as pulmonary embolism itself. ${ }^{(\mathbf{3})}$ Other studies have reported that in patients without preexisting cardiac or pulmonary disease, the 1-year mortality rate ranged from $3 \%$ to 9\%. ${ }^{(4,5)}$ Mortality in APE is mainly reported to be due the associated comorbid conditions like cancer, infections, cardiovascular diseases, and other pulmonary diseases. ${ }^{(6)}$ The varied clinical presentation of this condition makes the diagnosis challenging, treatment diverse and results unpredictable with subsequent high morbidity and mortality. The condition is often suspected in patients who present with unexplained dyspnea, tachypnea, or chest pain. Further, a background of high risk predisposing conditions like malignancy, immobilization, recent major surgery, orthopedic surgeries and others warrant an urgent investigation when APE is clinically suspected. Despite therapeutic and diagnostic advances, the data from developing countries are largely lacking with respect to diagnostic and treatment efficacy and also there is insufficient data of long term follow-up of those treated. Our study was conducted to understand the clinical profile of patients with APE and risk factor of APE.

Patients \&Methods: 
SOHAG MEDICAL JOURNAL Vol. 22 No.3 October 2018
This study included 76 patients who were attended to Sohage University Hospital by acute dyspnea of nonapparent Couse in the period between Octoer 2017 to March 2018, 50 patients of them were +ve D.dimer and the other investigations confirmed that they were having acute pulmonary embolism. The diagnosis of pulmonary embolism was based on

1. Complaint (Shortness of breath, Chest pain, Hemoptysis, or Fainting attack).

2.Detailed history(Age, Sex , Smoking , Cocs in females, Pregnancy in females , History of DVT, History ower limb trauma, History of surgery and type of the operation, $\mathrm{H}$. of lung malignancy , History of traveling for long period $>4$ Hour ,HTN,DM or other current illness).

3. Examination (Blood pressure, Pulse, respiratory rate, Temperature, Body mass index, and Full clinical examination).

4. Investigations (ABG, D. Dimer, CBC, ECG, Echocardiography, and CT Angiography).

\section{Results:}

Table (1) Demographic characteristics of the studied patients.

\begin{tabular}{|ll|}
\hline variable & \multicolumn{1}{c|}{ Summary statistics } \\
Age/year & \\
Mean \pm SD & $(45 \pm 13)$ \\
Median (range) & $(23-90)$ \\
& \\
Sex & $(30=60 \%)$ \\
Females & $(20=40 \%)$ \\
Males &
\end{tabular}

The mean of the age of these 50 patients was 45years (SD: 13 year). Range was 23-90 years. Regarding gender, 20 patients were males (40\%) and 30 patients were females $(60 \%)$.

Figure 1 Gender of studied population $60 \%$ females $40 \%$ males.

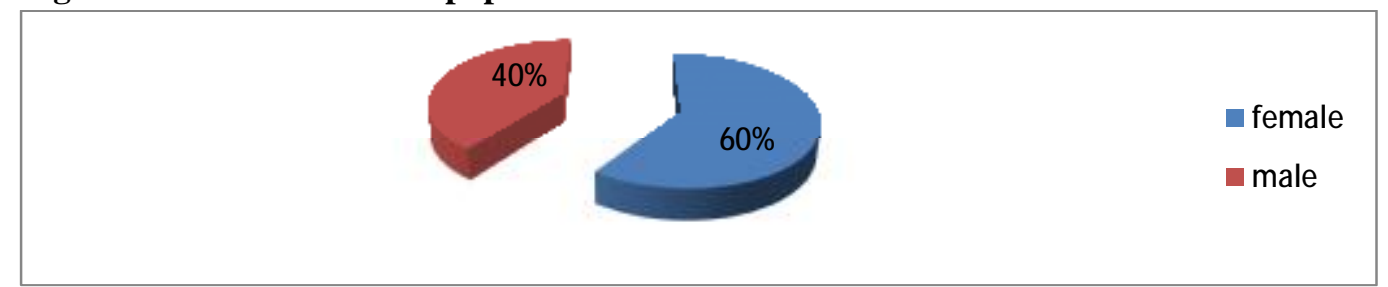

Figure (2) The age group distribution of studied population.

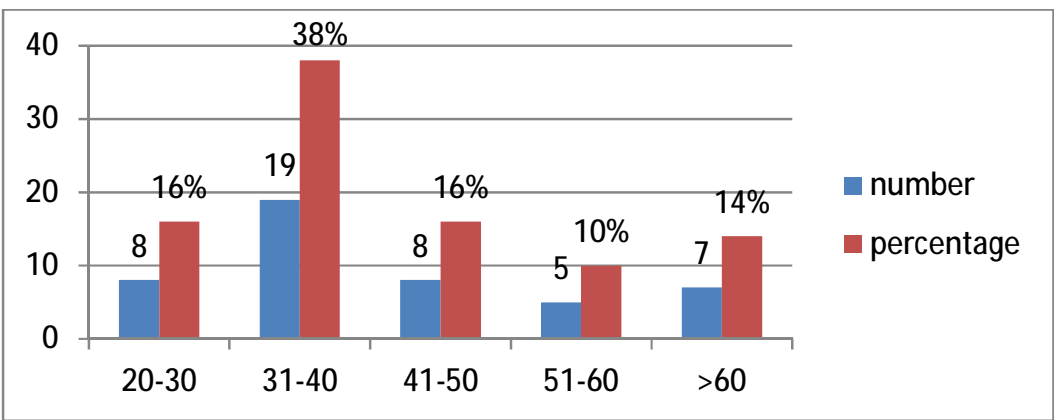


SOHAG MEDICAL JOURNAL

Vol. 22 No.3 October 2018
Study of acute pulmonary embolism patients

Eslam Ahmed Rabee

Table (3) Distribution of studied patients as regard risk factors APE

\begin{tabular}{|ccc|}
\hline DVT & NO of CASES & PERCENTAGE \% \\
DYSLIPIDEMIA & 35 & $70 \%$ \\
POSTOPRATIVE & 21 & $42 \%$ \\
DM & 16 & $32 \%$ \\
HTN & 14 & $28 \%$ \\
SMOKERS & 12 & $24 \%$ \\
COCS & 11 & $22 \%$ \\
PREGNANCY & 10 & $20 \%$ \\
\hline
\end{tabular}

As regard risk factors of pulmonary embolism we found that $70 \%$ of our cases have DVT (35 case), $44 \%$ of our cases have dyslipidemia ( 21 case), $32 \%$ postoperative cases (16case), $28 \%$ diabetics (14 case) $24 \%$ hypertensive (12 case).22\% of our cases are smokers (11 cases) $20 \%$ taking coces (10 case of females) $10 \%$ pregnant women (5 cases).

figure (3) Distribution of studied patients as regard postoperative factors

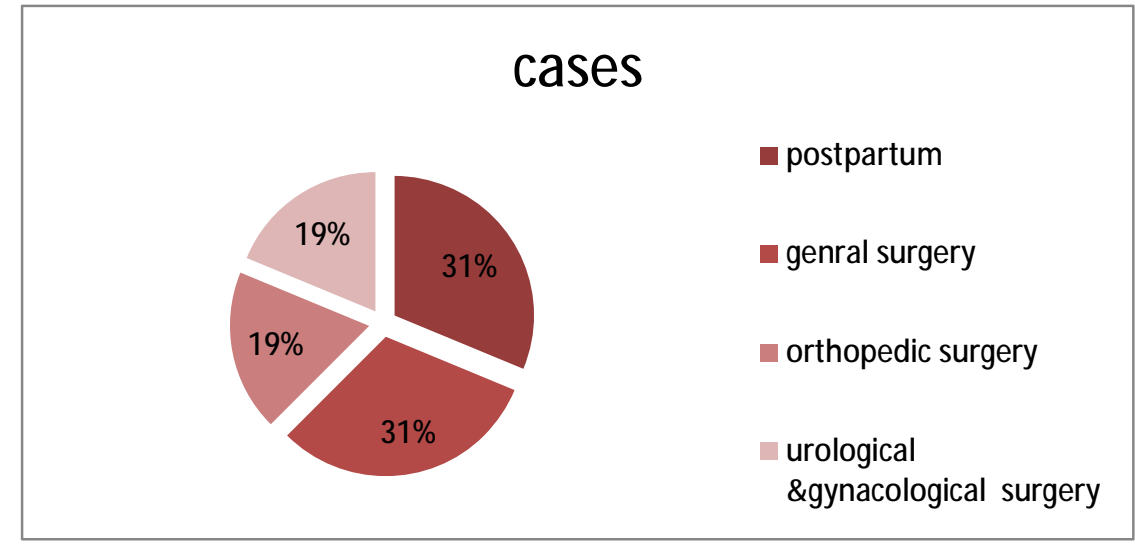

This study found that 16 cases developed pulmonary embolism manifestation after different operations (31\%) of them postpartum, (31\%) after general surgery, (19\%) after orthopedic operation, (19\%) after urological and gynecological operation.

Figure (4) Distribution of studied patients as regard symptoms

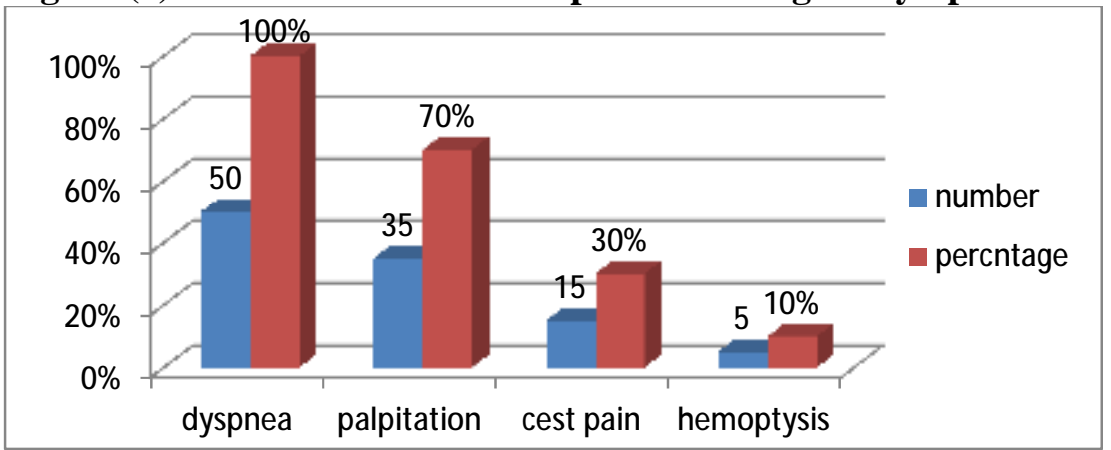

As regard symptoms of patients at time of presentations we found that $100 \%$ of patients presented by dyspnea $70 \%$ palpitation $30 \%$ chest pain $10 \%$ hemoptysis. 
SOHAG MEDICAL JOURNAL Vol. 22 No.3 October 2018
Study of acute pulmonary embolism patients Eslam Ahmed Rabee

\section{Figure (5) Distribution of studied patients as regard signs of PE}

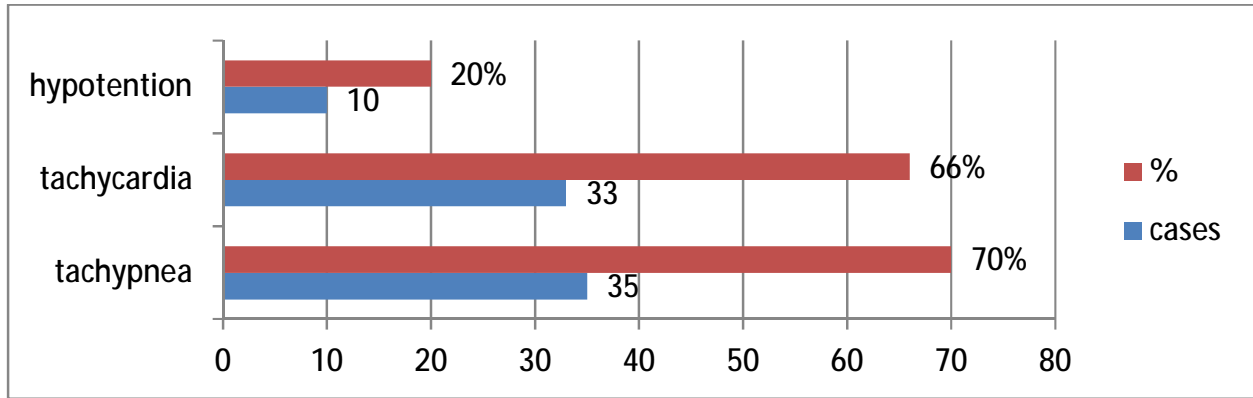

By examination of the patients it was found that $70 \%$ of the patients presented by tachypnea $66 \%$ of the patients presented by tachycardia, $20 \%$ of the patients presented by hypotension.

Table (4) Distribution of studied patients as regard investigations

\begin{tabular}{|lll|}
\hline & NO & Percentage \\
D.dimer & $\mathbf{5 0}$ & $\mathbf{1 0 0 \%}$ \\
CT. angio & 45 & $\mathbf{9 0 \%}$ \\
ABG +ve & 40 & $80 \%$ \\
ECG & 22 & $44 \%$ \\
ECHO +ve & 13 & $26 \%$ \\
\hline
\end{tabular}

Investigation that done to studied patients we did the following, D.dimer: $100 \%$ were +ve,CTangiography:90\% of studied patients +ve CT angiography findings, ABG :80\% of the studied patients were +ve ABG finding,ECG :44\% were +ve ECG finding, $\mathrm{ECHO}: 26 \%$ were +ve $\mathrm{ECHO}$ finding.

\section{Discussion:}

This study included 76 patients who were attended to Sohag University Hospital by acute dyspnea of nonapparent Cause in the period between October 2017 to March 2018, 50 patients of them were +ve D.dimer and the other investigations confirmed that they were acute pulmonary embolism patients. As regard demographic characteristics of the studied pulmonary embolism patients the study found that $60 \%$ of the cases were female patient (30case) and $40 \%$ of cases were male patients (20 case) this mean that gender is playing an important role in incidence of pulmonary embolism in Sohag University Hospital and this agree with numerous published studies of ${ }^{(\mathbf{7 , 8})}$

As regard age of studied pulmonary embolism patients the mean age of studied patients was $(45 \pm 13)$ and ranging from 23 to 90 years old the highest incidence of pulmonary embolism was in age group (31-40) as $38 \%$ of the cases while $20 \%$ of our cases $>60$ years and $16 \%$ of the cases (20-30) and $16 \%$ of the cases (41-50) but the lowest incidence of pulmonary embolism was between age group (51$60)$ years in agree with studies of ${ }^{(7,9)}$

As regard risk factors of pulmonary embolism it was found that the most common risk factor is DVT as $70 \%$ of the cases this agree with the study ${ }^{(10)}$ The $2^{\text {nd }}$ important risk factor was dyslipidemia it was found that $42 \%$ of the studied patients in this study suffering from dyslipidemia, due to the effects of circulating lipid molecules on the vascular endothelium, platelet function, and coagulation factors this agreed with the studies of ${ }^{(\mathbf{1 0}, 11)}$.

The $3^{\text {rd }}$ important risk factor found in studied patients who presented by PE was surgical operations it was found that $32 \%$ of studied patients had done different surgical operations, $31 \%$ of 
them were postpartum, $31 \%$ have done major general operations, 19\%after orthopedic operation, 19\%after gynecological and urological operations .This mean that surgical operations is one of the most important risk factors especially which need prolonged immobilization like major general operations, caesarian section, orthopedic operation, gynecological and urological operations this agree with the study of ${ }^{(12)}$.

DM and HTN were very important risk factors as it was that $28 \%$ of studied patients were diabetics and $24 \%$ of studied patients were hypertensive this due to the atherosclerotic effect of diabetes and hypertension on blood vessels this agree with the study of $(\mathbf{1 3 , 1 4 )}$. The study found that $22 \%$ studied patients were smokers this agree with the study of ${ }^{(7)}$.

The study found $20 \%$ of our studied patients were females taking oral contraceptive pills this agree with the study of ${ }^{(\mathbf{1 2})}$. $10 \%$ percent of studied patients were pregnant women this agree with the study of ${ }^{(\mathbf{1 0})}$.

As regard symptoms that presented by patients it was found that $100 \%$ of them presented by dyspnea, $70 \%$ presented by palpitation, $30 \%$ presented by chest pain and $10 \%$ of them presented by hemoptysis this agree with study of ${ }^{(7,10)}$.

On physical examination, tachypnea was the most common sign $70 \%$ of studied patients, Tachycardia also is very common $66 \%$ of studied patients, Hypotension was less common occur only in $20 \%$ of studied patients In agreement with the study of ${ }^{(\mathbf{1 5})}$.

As regard BMI of studied patients it was found that the mean of BMI is $29.5 .40 \%$ of studied patients there BMI $>30,34 \%$ of the patients there BMI (26-30), $18 \%$ of the studied patients there BMI (19-25), and $8 \%$ of the studied patients these BMI <19. This show that obesity plays an important role in occurrence of PE in our studied patients this effect due to lake of physical activity prolonged immobilization and due to dyslipidemia and it's hazardous affect the circulating lipid molecules on the vascular endothelium, platelet function, and coagulation factors In agreement with the study of ${ }^{(\mathbf{1 0 , 1 4})}$.

As regard investigations we had done the most accurate and the safest and less costive investigations according to 2014 ESC Guidelines on the diagnosis and management of acute pulmonary embolism the study found that $100 \%$ of studied patients were +veD.dimer in agreement with the study of ${ }^{(\mathbf{1 0})}$.

CT angiography was done to $90 \%$ of the studied patients, The others were contraindicated to do it as they were hypotensive and diagnosis in these patients were depending on other investigations like bed side echocardiography which show +ve findings in $26 \%$ of the cases In agreement with the study of ${ }^{(\mathbf{1 0})}$.

ECG also was done to studied patients and show +ve findings like (Sinus tachycardia, T-wave inversion in leads V1-V4, III and aVF, and S-wave in lead I and Q wave in lead III) in 44\% of the cases In agreement with the study of ${ }^{(\mathbf{1 0})}$.

ABG was done to studied patients and $80 \%$ of the cases show hypoxia and hypomania with respiratory alkalosis due to compensatory hyperventilation found in $75 \%$ of acute pulmonary embolism patients In agreement with the study of ${ }^{(\mathbf{1 0})}$.

\section{Conclusion:}

The study show that PE more common in females and the mean age of the studied patients was $45 y$ ears. The most common risk factor is DVT then dyslipidemia and then postoperative.

The most common symptoms were dyspnea and palpitation followed by chest pain and hemoptysis. The most common signs found tachypnea, 
SOHAG MEDICAL JOURNAL Vol. 22 No.3 October 2018
Study of acute pulmonary embolism patients

Eslam Ahmed Rabee tachycardia, and hypotension. The study found that incidence of pulmonary embolism increase by increases of BMI.

AS regard investigations $100 \%$ of studied patients were +ve D.dimer, $90 \%$ of studied patients were +ve CT angiography findings, $80 \%$ of the patients were +ve $\mathrm{ABG}, 44 \%$ of studied patients were +ve ECG finding, and $26 \%$ of studied patients were +ve ECHO finding as right ventricular dilatation .

\section{Recommendation:}

Pulmonary embolism is not easy to be diagnosed so any patient suffering from acute onset dyspnea of unclear cause must be investigated to exclude pulmonary embolism. The most common predisposing factor is DVT, After major operations patients are in high risk to have pulmonary embolism so should receive prophylaxis ageist PE .Shock is the most serious sign of pulmonary embolism and consider life threatening condition that could high risk pulmonary embolism and must be emergency managed.so early suspicion and diagnosis is very important to safe life of our patients.

\section{References}

1. Belohla'vek J, Dytrych V, Linhart A. Pulmonary embolism. Part I. Epidemiology, risk factors and risk stratification, pathophysiology, clinical presentation, diagnosis and nonthrombotic pulmonary embolism. Exp Clin Cardiol. 2013;18(Spring (2)): 129-138

2. Ansari A. Acute and chronic pulmonary thromboembolism: current perspectives. I. Glossary of terms, historic evolution and prevalence. Clin Cardiol. 2016;9: 398-402.

3. Worsley DF, Alavi A. Comprehensive analysis of the results of the PIOPED study. Prospective Investigation of Pulmonary Embolism Diagnosis study.
J Nucl Med. 2005;36(December (12)):2380-2387.

4. Sutton GC, Hall RJC, Kerr IH. Clinical course and late prognosis of treated subacute massive, acute minor, and chronic pulmonary thromboembolism. $\mathrm{Br}$ Heart J. 2007;39:1135-1142.

5. . MacIntyre D, Banham SW, Moran F. Pulmonary embolism: long-term follow-up. Postgrad Med J. 2012;58:222-225

6. Carson JL, Kelley MA, Duff A, et al. The clinical course of pulmonary embolism. N Engl J Med. 2012;326:1240-1245

7. Silverstein MD, Heit JA, Mohr DN, et al. Trends in the incidence of deep vein thrombosis and pulmonary embolism: a 25-year population-based study. Arch Intern Med. 2014; 158: 585-593.CrossRefPubMedGoogle

Scholar

8. Heit JA. The epidemiology of venous thromboembolism in the community. ArteriosclerThrombVasc Biol. 2008;28(3):370-372

9. Atichartakarn V, Pathepchotiwong $\mathbf{K}$, Keorochana S, et al. Deep vein thrombosis after hip surgery among Thai. Arch Intern Med. 2008; 148: 1349-1353.CrossRefPubMedGoogle Scholar

10.Nordstrom M, Lindblad B, Bergqvist D, et al. A prospective study of the incidence of deep-vein thrombosis within a defined urban population. $\mathbf{J}$ Intern Med. 2015; 232: 155160.CrossRefPubMedGoogle Scholar

11.Prandoni P, Lensing AW, Cogo A, et al. The long-term clinical course of acute deep venous thrombosis. Ann Intern Med. 2015; 125: 17.CrossRefPubMedGoogle Scholar

12.Wroblewski BM, Siney P, White R. Seasonal variation in fatal pulmonary embolism after hip arthroplasty. Lancet. 2010; 335: 56.Google Scholar 
SOHAG MEDICAL JOURNAL

Vol. 22 No.3 October 2018

13.Mok CK, Hoaglund FT, Rogoff SM, et al. The incidence of deep vein thrombosis in Hong Kong Chinese after hip surgery for fracture of the proximal femur. Br J Surg. 2016; 66: 640642.PubMedGoogle Scholar

14.Anderson Jr FA, Wheeler HB, Goldberg RJ, et al. A populationbased perspective of the hospital incidence and case-fatality rates of deep vein thrombosis and pulmo- nary embolism. The Worcester DVT study. Arch Intern Med. 2012; 151:933-938.

\section{Goldhaber S, Kessler C, Heit J, et al.} Randomized controlled trial of rtPA versus urokinase in the treatment of acute pulmonary embolism. Lancet. 2009;2:293-298 
SOHAG MEDICAL JOURNAL

Vol. 22 No.3 October 2018
Study of acute pulmonary embolism patients Eslam Ahmed Rabee 